\title{
Editorial
}

\section{Iterative Decoding and Cross-Layering Techniques for Multimedia Broadcasting and Communications}

\author{
Fred Daneshgaran, ${ }^{1}$ Massimiliano Laddomada, ${ }^{2}$ and Marina Mondin ${ }^{3}$ \\ ${ }^{1}$ Department of Electrical and Computer Engineering, School of Engineering, Computer Science and Technology, \\ California State University, Los Angeles, CA 90032, USA \\ ${ }^{2}$ Department of Electrical Engineering, Texas A\&M University, Texarkana, TX 75505, USA \\ ${ }^{3}$ Third School of Engineering - Information Technologies, Politecnico di Torino, 10129 Torino, Italy \\ Correspondence should be addressed to Massimiliano Laddomada, mladdomada@tamut.edu
}

Received 19 October 2008; Accepted 19 October 2008

Copyright () 2008 Fred Daneshgaran et al. This is an open access article distributed under the Creative Commons Attribution License, which permits unrestricted use, distribution, and reproduction in any medium, provided the original work is properly cited.

The explosive growth of multimedia applications over the Internet and the ever-increasing users' demands over commercial terrestrial digital multimedia broadcasting all over the world call for efficient physical and cross-layer techniques able to mitigate the potential problems limiting broadband services over wireless networks. In this scenario, mobile multimedia is expected to be one of the key services of future wireless mobile networks. Meanwhile, recent advances in digital communications have paved the way to a variety of standards aimed at providing multimedia services over terrestrial broadband networks. For example, DVB-H, TDVB, T-DMB, wireless LANs, and wireless MANs are some of the most recent standards enabling such technology.

Iterative decoding techniques for both source, channel, and joint source-channel coding and decoding and crosslayering techniques have proven to be very effective for providing a viable means of achieving capacity-approaching performance at very reduced computational burden.

This special issue is aimed at highlighting state-ofthe-art techniques on the most recent research advances enabling digital multimedia services over broadband wireless networks, focused on physical and cross-layering solutions.

The first paper "Lossy joint source-channel coding using raptor codes" by O. Y. Bursalioglu, M. Fresia, G. Caire, and H. V. Poor presents a joint source-channel coding scheme that combines the advantages and simplicity of entropy-coded quantization with the robustness of linear codes into a single linear encoding stage. Raptor codes are exploited in order to guarantee a continuum of coding rates with a single basic encoding algorithm.
The second paper "A simple scheme for belief propagation decoding of $\mathrm{BCH}$ and RS codes in multimedia transmissions" by Marco Baldi and Franco Chiaraluce proposes a new technique for decoding classic binary as well as nonbinary codes through the use of the belief propagation algorithm, which has proven to be effective in the decoding of low-density parity-check codes. The focus of the paper is on Reed-Solomon codes included in the CDMA2000 standard.

The third paper "Design of a VLSI decoder for partially structured LDPC codes" by Fabrizio Vacca, Libero Dinoi, and Guido Masera focuses on the development of a new class of partially structured low-density parity-check codes, and discusses the design of the VLSI decoder, as well as a novel decoding technique called split decoding.

The fourth paper "Turbo decoder for low power ultrawideband communication systems" by Esam Obiedat, and Lei Cao proposes a new method to reduce the computational complexity of turbo decoding in ultra-wideband systems employing the orthogonal frequency division multiplexing (OFDM) modulation. A bit-level stopping technique based on the constrained decoding method is presented with the aim of detecting code convergence on each OFDM subcarrier.

The fifth paper "System level evaluation of innovative coded MIMO-OFDM systems for broadcasting digital TV," by Youssef Nasser, Jean-Francois Helard, and Matthieu Crussière investigates the application of space-time codes in single frequency networks for digital TV broadcasting. A 3D space-time-space block code is proposed in the context of 
the future terrestrial digital TV systems using coded MIMOOFDM techniques.

The sixth paper "Stationary interference cancellation in upstream coordinated DSL using a turbo-MMSE receiver" by Issam Wahibi, Meryem Ouzzif, Jérôme Le Masson, and Samir Saoudi investigates the use of stationary interference cancellation in upstream digital subscriber lines (DSLs) employing turbo-MMSE techniques. The use of noise whitening techniques coupled with a mean-squared error iterative receiver is proposed in order to approach the matched filter bound of a DSL-coordinated system.

The seventh paper "Iterative Mean Removal Superimposed Training for SISO and MIMO Channel Estimation" by Omar Humberto Longoria-Gandara, Ramón Parra-Michel, Luis Miguel Bazdresch, and Aldo Orozco describes a novel iterative radio channel estimation algorithm based on a superimposed training estimation technique. The proposed algorithm is well tailored to SISO as well as MIMO channels.

The eighth paper "Block iterative/adaptive frequencydomain channel estimation for cyclic-prefixed single-carrier broadband wireless systems" by Jong-Seob Baek and JongSoo Seo presents a new block iterative/adaptive frequencydomain channel estimation algorithm. The idea consists in iteratively estimating the frequency response of the channel by employing a weighted element-wise block adaptive frequency-domain channel estimation scheme that relies on the soft information obtained by a soft-input soft-output decoder.

The last paper of this special issue "Real time communications in large scale wireless networks" by Liang Song and Dimitrios Hatzinakos investigates the use of largescale cognitive networking methods in multihop wireless networks. Based on the proposed method, data packets travel along opportunistic paths using opportunistically available spectrum in every hop. The proposed algorithm is key to guaranteeing quality of service in wireless mesh networks for broadband networks supporting multimedia services, and visual sensor networks for surveillance.

\section{ACKNOWLEDGMENTS}

The authors are grateful to the reviewers for their invaluable work and to the authors of the papers collected in this special issue.

Fred Daneshgaran Massimiliano Laddomada Marina Mondin 

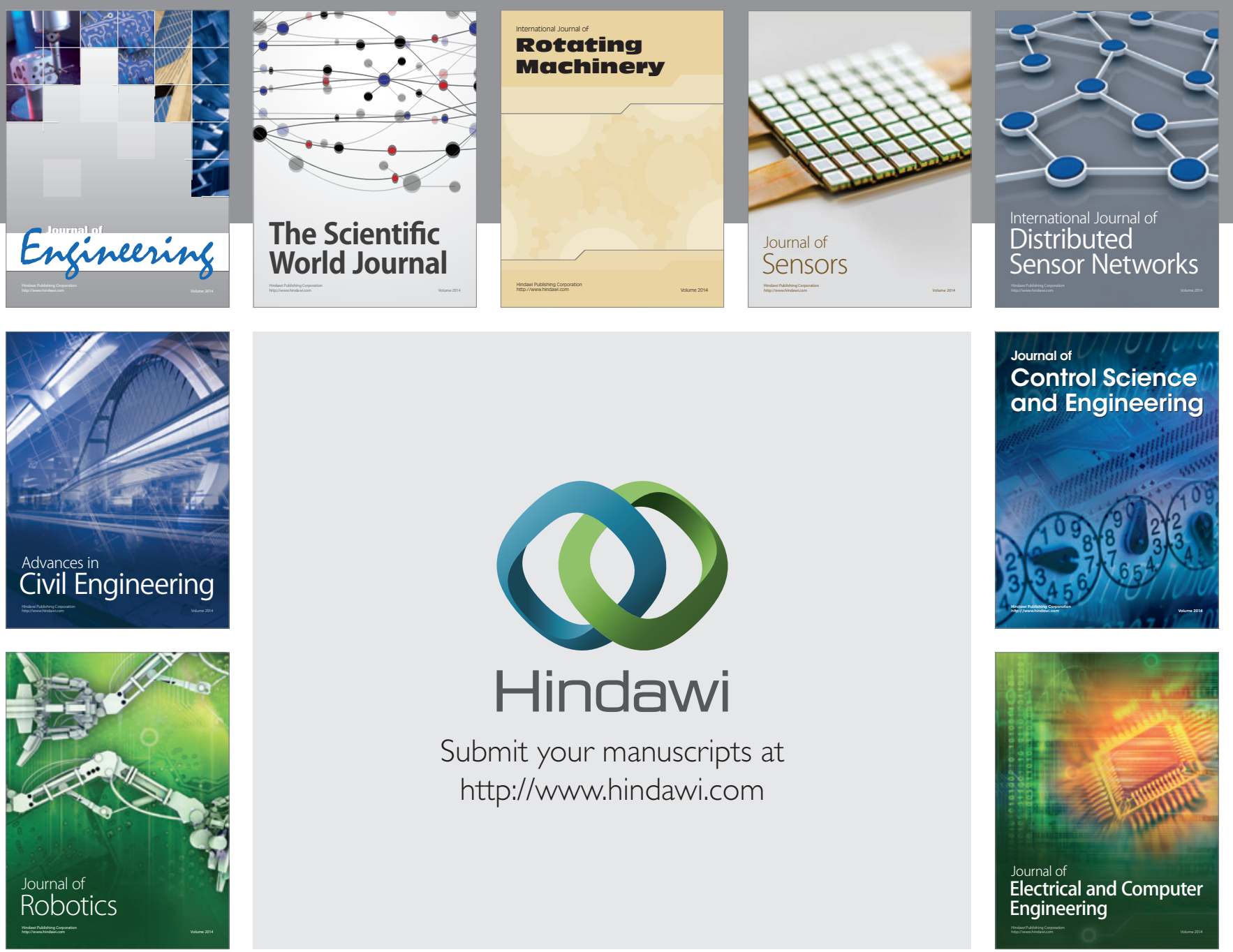

Submit your manuscripts at

http://www.hindawi.com
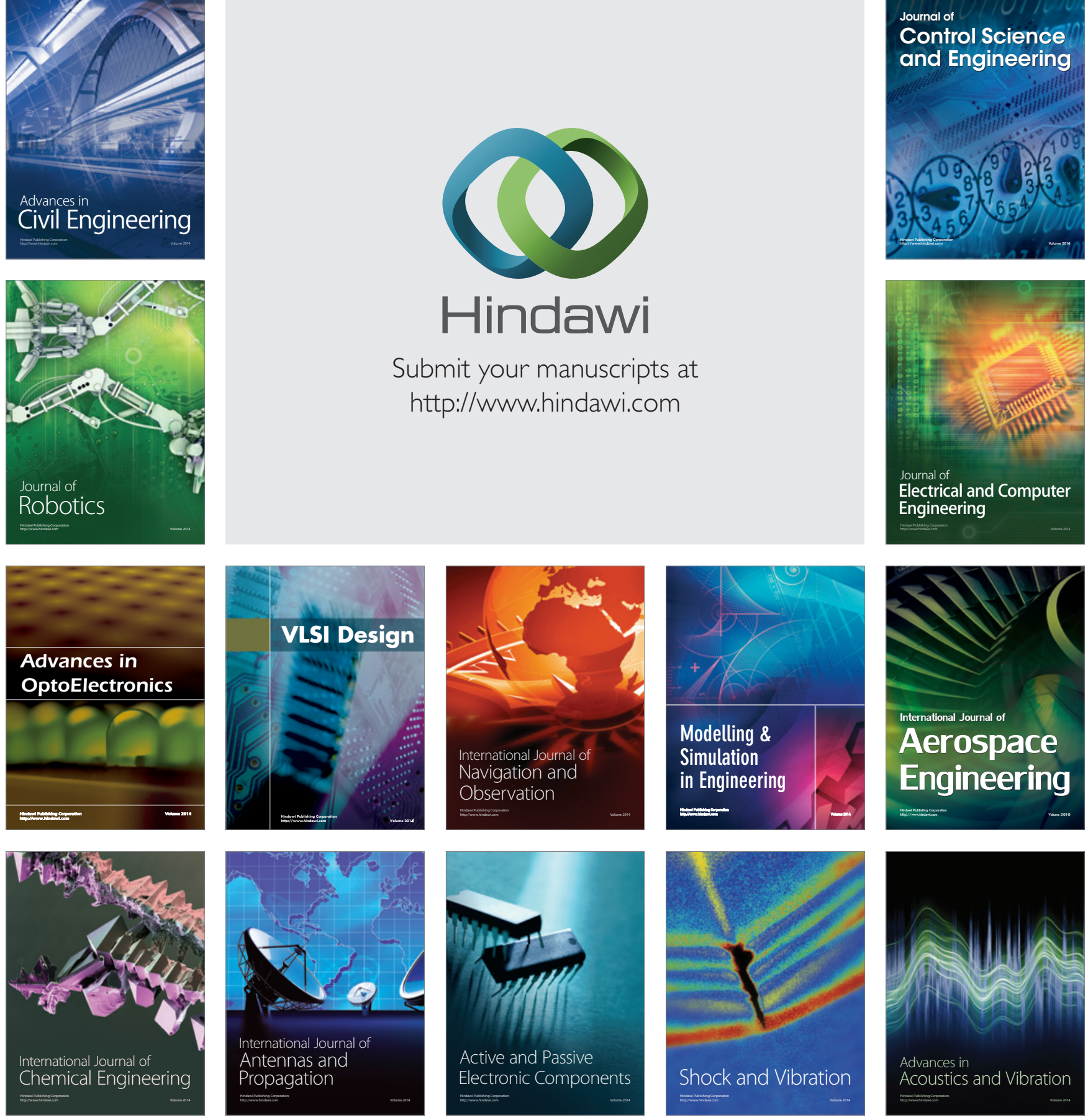